\title{
Analysis of Interface and Screen for Ground Control System
}

\author{
Eunghyun Lee1, Suhwan Kim², Yongjin James Kwon ${ }^{*}$ \\ ${ }^{1}$ Department of Industrial Engineering, Ajou University, Suwon, South Korea \\ ${ }^{2} 1^{\text {st }}$ Division, $3^{\text {rd }}$ Department, Agency for Defense Development, Daejeon, South Korea \\ Email: *yk73@ajou.ac.kr
}

Received 10 May 2016; accepted 19 May 2016; published 26 May 2016

\begin{abstract}
This study is a preparation phase for visualization of utilized information using ergonomic user interface and standardization of elements for GCS (Ground Control System). Therefore, we investigated the instances of GCS (such as hawk and patriot missile's GCS) for defense system. Based on the collected data, we compared and analyzed the GCS screen design. In this paper, we conduct case study for ergonomically development of GCS. It is expected that this research improves the situational awareness and reduces the user's task load.
\end{abstract}

\section{Keywords}

\section{Ground Control Station, Human Interface, Situational Awareness, Task Load}

\section{Introduction}

The Hawk missile is an air guided weapon that incapacitates guided missiles and enemy aircrafts that invade from low or medium altitudes. It was first invented in the United States in 1953, and in 1964, it was improved by installing central digital data processing equipment at the command post, which automatically performs important procedures, such as target process, threat order decision making, and the confirmation of target interceptions. Generally, one unit of shots, which is 3 shots, is installed on the launcher. Due to the fact that the launcher can be easily transported with a helicopter or a vehicle, it is used for position defense on the fields. Also, it is appropriate for shooting down enemies that are invading from low altitudes because its lowest interception altitude is $30 \mathrm{~m}$. MIM-23A, the most basic form of the Hawk missile, and MIM-23B were deployed in 1960 and 1973 respectively, and over 20,000 units exist in the world currently [1] [2]. Through continuous improvements, the Hawk XXI was developed; the Hawk XXI is able to launch both on the ground and on the sea and attack targets on the ground and on the sea within 124 miles. Hawk XXI is an updated version of the Hawk missile that has eidetic HMI (Human-Machine Interface) and FDC (Fire Distribution Center) [3]. Having replaced Nike Hercules and the Hawk system, it has various functions, such as communications, command and control, radar surveillance, and missile guidance [4]. PAC-1 and PAC-2, which are the early versions, uses indirect explosion method, while PAC-3 uses pinpoint strike. The Patriot missile system is an air defense system, which can be

\footnotetext{
${ }^{*}$ Corresponding author.
} 
used for long-distance, all altitudes, and under all types of weather, developed in order to intercept ballistic missiles, cruise missiles, and cutting-edge missiles of the enemy [5]. Its identifier code is MIN-104, and it was developed in collaboration with Raytheon Missile, Air Defense Division, and Martin Marietta. The Patriot system uses multi-function C-band phased array radars, and can perform various functions, such as thorough surveillance, target detection, target racing, and leading missiles. The ECS (Engagement Control Station) is a general control center of the Patriot corps, and the ICC (Information Coordination Central) controls the Patriot system battalion by battalion. The ICC efficiently supervises air battles by controlling integrated air defense weapons, such as the Hawk and the Patriot. Also, the ICC, one of the main components of the Patriot FDC (Fire Direction Center), controls the Patriot missile launches and delivers information about battlefield situations to JTIDS (Joint Tactical Information Distribution System) or MIDS (Multifunctional Information Distribution System) [6].

\section{Case Study}

\subsection{System Overview}

Most functions and system settings of the Hawk Missile GCS are operated by using the switches near the radar screen. The picture 2 shows the old version and newest XXI version of the Hawk Missile GCS. As mentioned before, the Hawk Missile system, developed in 1953, is currently being operated after numerous improvements; however, the operators have had many difficulties with performing their tasks with this system because of its low resolution screen, non-intuitive buttons, and narrow working space. And the Hawk XXI missile GCS, which is currently used in South Korea. As you can see in Figure 1 Hawk XXI GCS has a completely different layout from that of the old version. Not only do most control and operation are done on the touch screen, but it also has a high resolution monitor, which enables the operators to clearly observe battlefield situations. On the old and newest Patriot as shown below Figure 2 anti-aircraft defense system, different types of control indicator consoles are located near the radar screen. Even though the size of the display of the Hawk increased, it still has a low-resolution display and an extremely small color palette, which hinders the situation reading ability of the operator. On the other hand, the console of the new version of the Patriot (PAC-3) was improved from the old console with new technology. MMS (Modern Man Station), the name of the console of PAC-3, has two 30-inch touch screens arranged on top of each other, which improves the operator's clear battle field situation observation, precise decision making, and accurate command order.

\subsection{Radar Screen and User Interface}

\subsubsection{Hawk Missile}

The operator can perform various tasks, such as target detection, target identification, threat order decision making, and target assignment. Figure 3 below shows the radar screen of the old and newest Hawk missile



Figure 1. Hawk missile GCS (Old type; XXI). 




Figure 2. Patriot GCS (Old type(PAC-1,2); PAC-3).

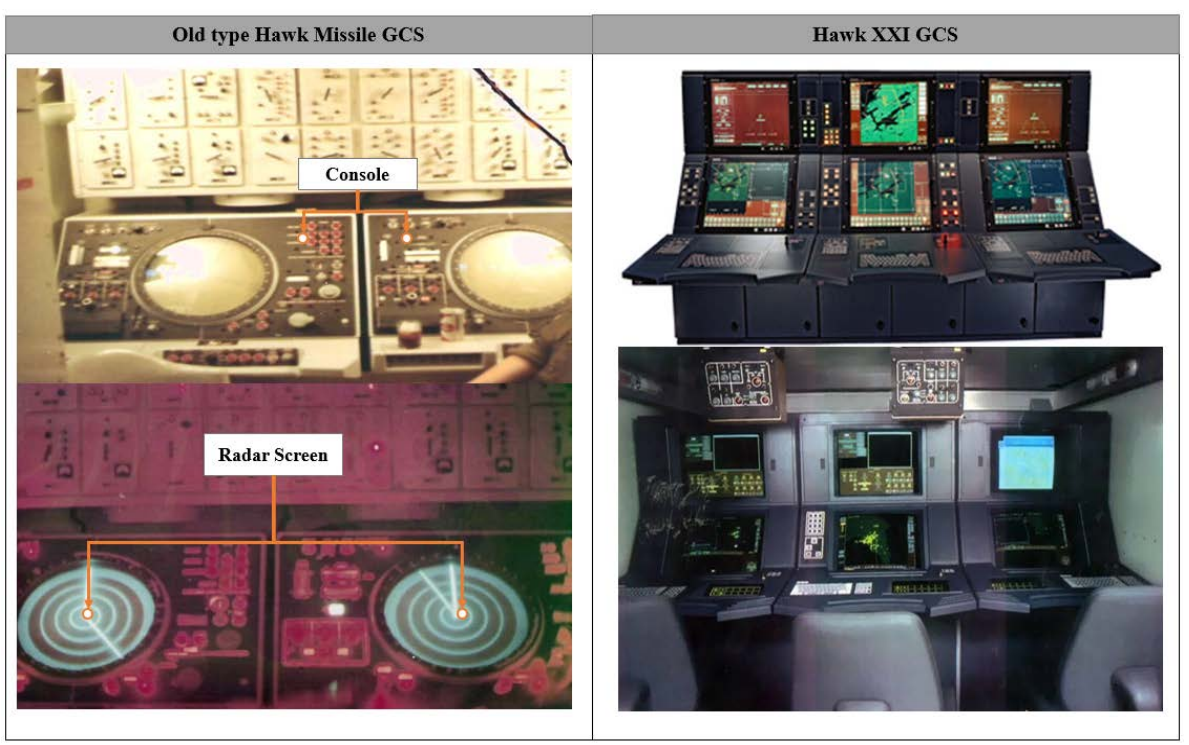

Figure 3. Hawk missile consoles and radar screen.

consoles. In the case of old type of hawk's radar screen is located in the center of the Console in a circular shape. It has a black background and several colors that help deliver information to the operator. However it does not have a tactical map that helps the operator see the battlefield situation. The radar screen is operated by using the knobs which are located on each side of the screen. On the other hand, FDC, the new version of Hawk XXI Missile Console is able to perform thorough distributed operation. It is also an open type SW/HW architecture built based on flexibility, expandability, and interoperability, which enables easy additions of new technologies and functions. The characteristics of FDC include real-time data processing, interoperability, intuitive real-time air defense combat order, and HMI that provides more precise and accurate target attacks. Due to its wide range of uses and flexibility, one FDC could be used on several different programs. The operator uses two 20 inch screens that are placed on top of each other. The bottom screen is a touch display which also lets the operator to choose and see different layouts. The system is operated based on a 2D tactical map with different combinations of colors. 


\subsubsection{Patriot Missile}

MIM-104A Patriot Missile, which is the standard model of the Patriot Missile deployed in 1982, was an antiaircraft missile that was originally designed for the aircraft interception purpose only like Hawk and Nike. In 1986, some parts of MIM-104A were upgraded. However, as the number of tactical ballistic missiles increased, the United States Armed Forces felt the need to prepare for this trend and started taking action. In order to fight against tactical ballistic missiles, they have been upgrading the software of the Patriot missile since 1985 and also have strengthened the ability to fight against ECMs (Electronic Counter Measures). Additionally, they have improved the software that affects the detection/chase algorithm such that the angle of climb of a multi-function phased array radar is raised from 45 to 90 degrees to detect and chase. With these improvements, the Patriot Missile successfully intercepted, which is a short-distance ballistic missile, in its test run in September, 1986. This signified that the United States now had the ability to fight against anti-tactical ballistic missiles. Having made continuous improvements on the Patriot since 1983, they have used the MIM-104B Patriot, which is Stand-Off Jammer Countermeasures, since 1988. This upgraded version is called PAC-1 (Patriot Advanced Capability Level 1). However, PAC-1's lack of destructive power during ballistic missile interceptions have led to a lot of problems, such as causing damage on the ground with the debris of the airframe created from not destroying the ballistic missiles completely. As a solution to this problem, they changed some combat software to PDB-3 and incorporated MIM-104C, also known as PAC-2, which intercepts tactical ballistic missiles, into the system. PAC-2 enables warhead to explode when it is in most contact with the ballistic missile. The display screen and the control system of PAC-1 and PAC-2 are controlled by two operators.

For the old version of the Patriot air defense system, a lot of different control switches are located around the radar screen, as shown in Figure 4. The switches give information about current conditions by lighting up the switches of the functions that are in operation. The radar screen of the Patriot has a circular shape in the center of the console. It indicates information in white on the black background, which show targets and objects in the shooting range of the Patriot. The old version of the Patriot does not use different colors to indicate allies or enemies and other conditions, which causes the operator to have problems reading the information especially when there is a lot of objects. On the other hand, PAC-3 has the same interface as PAC- 1 and 2 . Therefore, the operators who are already familiar with the regular system do not need to be trained to use PAC-3, as shown in Figure 4 below.

The display screen of PAC-3 lets the operator choose different functions according to their needs. The color coordination for determining allies or enemies increases the ability of situation awareness. The buttons that used to operate the regular system are all located on the 30-inch touchscreen, and each button has a different color to indicate the condition of each function.

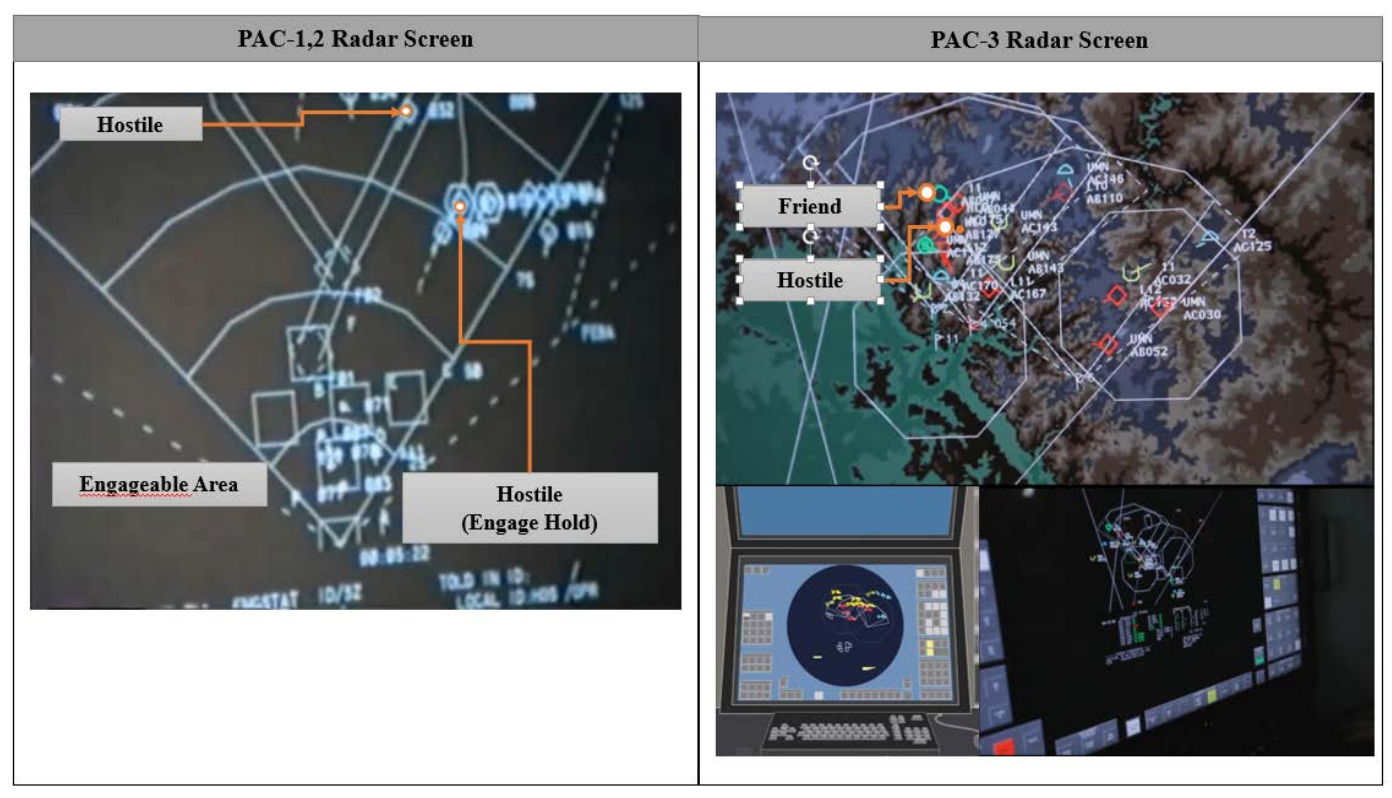

Figure 4. PAC-1, 2 radar screen and PAC-3 console/radar screen. 


\section{Results}

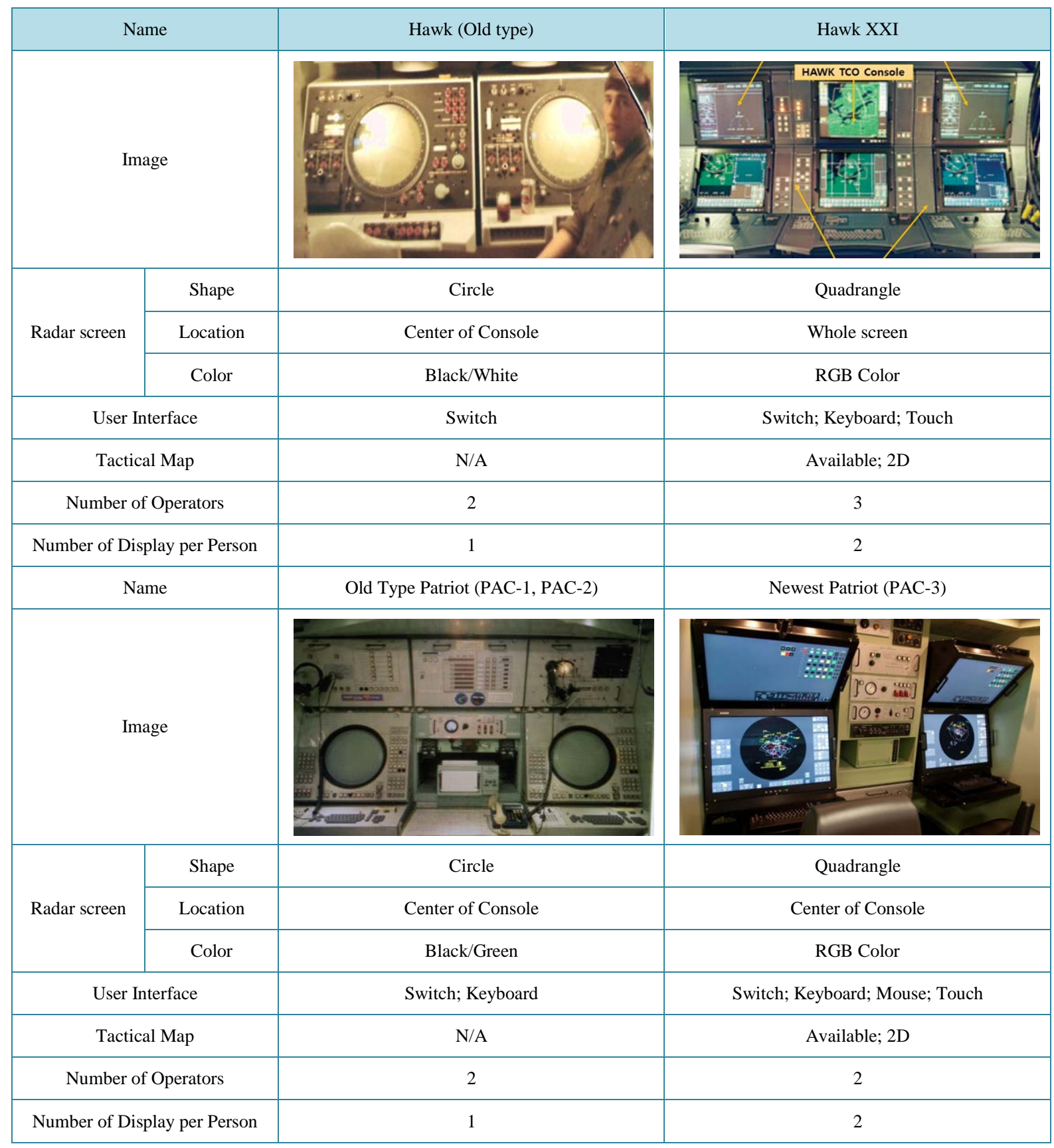

\section{Conclusion}

The old version of anti-air missile consoles are equipped with a single circular CRT (Cathode Ray Tube) screen for each operator. The CRT display had a lot of problems, such as its big size and a small color palette, which hindered the operator's ability to read the information. However, the new version of anti-air missile consoles are equipped with LCD displays and two touch screens for each operator. According to their needs, the operator can change the layout of the display to see the situation conveniently. Not having to look around, the operator is able to focus on the information on the touch screen, which results in better situation awareness and fast decision making. The new version also has a tactical map, which is a critical component in increasing the mission success rate. 


\section{Acknowledgements}

This work was supported by the Agency for Defense Development (ADD) under the Contract No. UD140066CD. The authors wish to express sincere gratitude for the financial support.

\section{References}

[1] Olmos, O., Wickens, C.D. and Chudy, A. (2014) Tactical Displays for Combat Awareness: An Examination of Dimensionality and Frame of Reference Concepts and the Application of Cognitive Engineering. The International Journal of Aviation Psychology, 10, 247-271. http://dx.doi.org/10.1207/S15327108IJAP1003_03

[2] Brown, C., Fagan, P., Hepplewhite, A., Irving, B., Lane, D. and Squire, E. (2014) Real-Time Decision Support for the Anti-Air Warfare Commander. Proc. 6th Int. Command Control Res. Technol. Symp. (ICCRTS), 2001.

[3] Filippidis, A., Blandford, S., Foster, K. and Moran, G. (2006) Simulation Activities Using Gateway and Tactical Digital Information Links. Published by Defence Science and Technology Organisation, 1-13.

[4] Lee, K. and Lee, H. (2009) Development Strategies of Anti-Aircraft Weapons 1. National Defense and Technology, 30-37.

[5] Lee, K. and Lee, H. (2009) Development Strategies of Anti-Aircraft Weapons 2. National Defense and Technology, 74-81.

[6] Lee, J.H.E., Kim, D., Kwon, Y. and Kim, S. (2015) A Analysis of the User Interface for the Russian Air Defense Weapon Systems. Korean Institute of Industrial Engineer, 417-434. 